\title{
Análisis de citación de la revista Ciência da Informação del Ibict
}

\author{
Adilson Luiz Pinto \\ Doctorando en documentación. Universidad Carlos III de Madrid. \\ Email: aluiz@ceaes.uc3m.es \\ Beatriz-Ainhize Rodríguez Barquín
Doctoranda en documentación. Universidad Carlos III de Madrid.
E-mail: beatriz.rodriguez@bib.uc3m.es-ainhize@inf.ie.uc3m.com
}

José Antonio Moreiro González

Decano de la Universidad Carlos III de Madrid.

E-mail: jamore@bib.uc3m.es

\section{Resumen}

Esta investigación estudia el análisis de citaciones utilizadas por los autores que publican dentro de la revista Ciência da Informação, de donde fueron recuperados 278 artículos científicos en el periodo de 1995 a 2003. En relación al método, utilizamos un fichero en Excel para cuantificar los datos, donde dividimos por género de citas bibliográficas. Se han aplicado técnicas cuantitativas de investigación para analizar las citas de las revistas utilizadas, trabajando con el factor de impacto de las revistas ISI y algunas del SciELO. Otro aspecto fue analizar las citas de libros, tesis, actas de congresos, documentos electrónicos y comunicaciones, que son una fuente fundamental en la confección de un trabajo científico. Posteriormente se investigaron las relaciones y la colaboración institucional de los autores que publican dentro de la revista Ciência da Informação, utilizando el programa Pajek. Y por fin observamos la relación entre las palabras-clave empleadas por los autores que publicaron en la revista, con las temáticas de revistas indizadas en ISI, SciELO y Latindex. Para las conclusiones, destacamos que el mejor periodo de las citas utilizadas fue de 1996 hasta 1998, con una fuerte presencia de revistas ISI y de la propia revista Ciência da Informação.

Palabras-clave

Análisis de citación. Revista Ciência da Informação - Ibict. Revistas ISI. Revistas SciELO y Latindex.

\section{Citation analysis of the Ciência da Informação journal of the Ibict}

\section{Abstract}

The research studies the analysis of used citations by authors who publish inside the Ciência da Informação journal, where were rescued 278 scientific papers between the period 1995 to 2003. Related to the method, an Excel file was used with the aim of quantify the data, and where we divide by genre the bibliographic cites. Quantitative methods of investigation have been applied to analyze the cites of the used journals, working with the impact factors of ISI journals and some of SciELO. Another aspect of the present study was to analyze the books citations, thesis, electronic acts of congresses, electronic documents and communications, which are a fundamental source to make a scientific work. Lately, the relations and de institutional collaboration of authors who publish inside the Ciência da Informação journal were investigated, using Pajek program. Finally we observed the relation between the used keywords by the authors which published in the journal, with the indexed thematic in ISI, Scielo an Latindex. To conclude, we emphasized that the best period of cites used was between 1996 until 1998, with a strong presence of ISI journals and the own Ciencia da informaçao journal.

\section{Keywords}

Citation analysis. Ciência da Informação Journal - Ibict. ISI Journals. SciELO and Latindex Journals.

\section{INTRODUCCIÓN}

Desde la publicación del Journal des Sçavans -editado en Francia por Denis de Sallo, y de las Philosophical Transactions: giving some Accompot of the present Undertakings, Studies and Labours of the Ingenious in many considerale parts of the World, por la Royal Society británica (Siglo XVII) los periódicos científicos pasan a ser incorporados en la comunidad pensante (BIOJONE, 2001).

En Brasil esta iniciativa surge con la creación de Gaceta Médica de Río de Janeiro, de 1862, y posteriormente la Gaceta Médica de Bahía de 1866.

En los días actuales se estima que se publican más de 600 mil revistas científicas en todo el mundo, se estima que para alimentarlas son necesarios entre seis y siete millones de artículos científicos (TRZESNIAK, 2001). Tal proliferación de títulos trae subyacente un conjunto de problemas. La evaluación sobre la calidad de los artículos muestra que un 50\% de ellos contienen errores estadísticos; y datos sobre el impacto indican que aproximadamente un 50\% de los artículos publicados jamás llegan a ser citados o siquiera consultados (TRZESNIAK, 2001). Además de la deficiente calidad de los artículos propiamente dichos, la irregularidad en la publicación y distribución, la ausencia o deficiencia en la normalización de los artículos y de la revista, la ausencia de aceptación de indización en bases internacionales reconocidas y otras cuestiones vinculadas a la lengua son aspectos que merecen la atención de la comunidad científica (FERREIRA, 2001).

Algunas iniciativas en este sentido en América Latina son el proyecto Latindex ${ }^{*}$ y SciELO**, acciones que

\footnotetext{
* Sistema regional de información en línea para revistas científicas de América Latina, el Caribe, España y Portugal.

** La Scientific Eletronic Library Online - SciELO es una biblioteca electrónica que comprende una colección seleccionada de las revistas científicos brasileños. La SciELO es el resultado de un proyecto de búsqueda de la FAPESP - Fundación de Amparo a la Búsqueda del Estado de San Paulo, en asociación con La BIREME - Centro LatinoAmericano y de Caribe de Información en Ciencias de la Salud. El partir de 2002, el Proyecto cuenta con el apoyo del CNPq - Consejo Nacional de Desarrollo Científico y Tecnológico. El Proyecto tiene por objetivo el desarrollo de una metodología común para la preparación, almacenamiento, diseminación y evaluación de la producción científica en formato electrónico. Con el avance de las actividades del proyecto, nuevos títulos de las revistas están siendo incorporados a la colección de la biblioteca.
} 
trabajan en enfocar y normalizar las principales revistas del continente y donde está indizado nuestro objeto de investigación, la revista Ciência da Informação.

En relación a la importancia de la revista Ciência da Informação, es fundamental hacer una revisión literaria de este medio de comunicación científica, donde en 1972 el Instituto Brasileño de Información Científica y Tecnológica (Ibict) lanza su revista científica (Ciência da Informação), siendo la primera en América Latina que se centró en discutir problemas de generación, control y transferencia de información y otros temas que fueron de constante importancia para la evolución de la ciencia.

El periódico celebró su treinta cumpleaños en 2003, y entre sus características destaca su sólida producción y puntualidad en sus ediciones, con investigaciones actuales. Siendo recurrida en los principales servicios de indización de América Latina y Europa, como el Latindex, el SciELO, el Library and Information Science Abstracts (LISA) ${ }^{*}$ y el Francis ${ }^{* *}$, cumpliendo su papel de divulgadora del conocimiento generado en territorio nacional e internacional.

La Revista Ciência da Informação ha sido, durante estos años, el canal de comunicación entre la producción técnico-científica en ciencias de la información, en campos correlatos para la información en ciencia e tecnología, diseminando investigaciones y estudios bajo la forma de artículos, comunicaciones y relatos empíricos. Aunque editada por el Ibict, la revista abre sus páginas a los profesionales de información de diversas instituciones, como medio de diseminación reconocido por la comunidad del área, fiel a su principio interdisciplinario y coherente con la naturaleza del área de su especialización (PINHEIRO, 1996).

En 2001, Mueller y Pecegueiro identificaron la revista en la década de 1990, evaluando el índice de autoría, los orígenes institucionales de los autores que producen en la revista, la frecuencia de los artículos por temáticas y una aplicación de los indicadores por año.

Posteriormente, fue relatado un estudio sobre la importancia de la revista en el ámbito científico

\footnotetext{
* Base de datos sobre Biblioteconomía, Documentación, Archivística, Gestión del Conocimiento y Tecnología de la Información, que corresponde una cobertura espacial a partir de 1969 hasta los días actuales.

${ }^{* *}$ Base de datos francesa del Centre National de la Recherche Scientifique, cuya literatura se basa en los estudios de las Humanidades, y el control se realiza por el Institut de L'Information Scientífique et Technique (INIST).
}

brasileño, describiendo los principales indicadores de la revista, con datos de los editores de la revista, las características de la revista, las temáticas de los artículos, el índice de autoría, autores más productivos, contribución por autores y por regiones entre otros (PINHEIRO; BRÄSCHER; BURNIER, 2005).

A partir de estos dos estudios, planteamos un análisis científico de las citaciones dentro de la revistas Ciência da Informação, siendo un contra-punto, puesto que la parte de la autoría y de la representación científica ya fue desarrollada por Mueller y Pecegueiro y por Pinheiro, Bräscher y Burnier.

Partiendo de esta visión, nuestro objetivo principal está centrado en analizar las características de las citaciones utilizadas por los autores que publican en la revista Ciência da Informação durante el periodo de 1995 hasta 2003.

En relación a los objetivos específicos, planteamos: (i) Analizar los autores más citados en el periodo; (ii) Estudiar las revistas más citadas, para diagnosticar cuáles son los parámetros editoriales de los científicos que publican en la revista Ciência da Informação; (iii) Identificar el factor de impacto de las revistas indizadas en ISI y en SciELO; (iv) Evaluar el origen (país) y año de las tipologías estudiadas*, para poder medir qué años tuvieron un número mayor de publicaciones; (v) realizar un estudio de redes sociales entre los autores que publican en la revista, teniendo en cuenta cuáles son las instituciones que colaboran para publicar dentro del periódico; (vi) concluir los análisis con un estudio de relación de palabras-clave con las citas utilizadas por los autores, para averiguar si las temáticas de los artículos publicados en la revista Ciência da Informação se corresponden con el área temática de las citas utilizadas, utilizando las listas de temáticas del Journal Report Citation (para ISI), informaciones de las demás citas en sus sitio web (para las demás revistas) y relaciones con los títulos de las tipologías (libros; tesis; actas de congreso e etc).

\section{MÉTODO}

Este trabajo analizó un total de 278 artículos científicos (editados entre los años de 1995 hasta 2003) en lengua portuguesa, española e inglesa. Los demás tipos de documentos como las comunicaciones y relatos empíricos existentes en las ediciones de la revista, no fueron utilizados.

\footnotetext{
* Las demás tipologías (Libros; Actas de Congreso; Comunicaciones; Documentos Electrónicos y Tesis) y los países que hacen parte esos tipos de materiales bibliográficos.
} 
La división de las tipologías fue realizada en libros; Actas de Congresos; Comunicaciones; Documentos electrónicos; Tesis, y para la verificación de la visibilidad de las citas en artículos de periódicos dividimos en revistas indizadas en la base de datos del ISI* y en las demás bases de datos, priorizando verificar el factor de impacto de las revistas.

La parte gráfica fue confeccionada a través de una planilla en Excel, cita por cita, debido a que el formato de recuperación es en $p d f$.

Para identificar mejor el trabajo, representamos las técnicas y acciones utilizadas en la configuración de este trabajo, donde dividimos en:

- Cuantificación de autores (BRADFORD, 1948), seguido de división por tipología (MACIASCHAPULA, 1998) y por un ranking de autores con mayor número de citas (GARFIELD, 1972) y (WHITE \& McCAIN, 1989);

- Fueron también divididas las citas de revistas por categorías, denominadas ISI y otras revistas no ISI, para poder recuperar el factor impacto** (GARFIELD, $1994 \mathrm{y}$ 1996) fue utilizado el factor de 2002 por ser el año en el que las revistas que poseen este genero tienen sus mayores picos de impacto, tanto en ISI como en SciELO;

- Fueron separadas las citas de actas de congreso y libros por año (ZANOTTO, 1999) verificando el empleo de literaturas nacionales y extranjeras por los autores;

- Una vez obtenidos los datos anteriores, (i) se elaboró una relación entre las palabras-clave con las categorías de áreas en el ISI, la cuál también (ii) se enfocó a las relaciones instituciones que publican dentro de la revista (MOYA-ANEGÓN, 2004), donde fueron visualizadas las áreas temáticas por las informaciones dadas en el sitio de cada revista. Para este tipo de investigación fue utilizado el programa Pajek ${ }^{* * *}$ para representar las publicaciones en conjunto (FAZITO, 2002) y la

\footnotetext{
- En esta análisis también están contempladas las revistas de la base del conocimiento SciELO, que utiliza la misma técnica de generar factor de impacto que el utilizado por ISI.

- El valor del factor de impacto es obtenido dividiendo el numero total de citaciones de los artículos acumulados en los últimos dos años por el total acumulado de artículos publicados por la revista en lo mismo período. Actualmente las revistas indizadas en el ISI son consideradas para el calculo del factor de impacto internacional, pero existe también una iniciativa del Programa SciELO, que empezó recientemente a calcular los factores de impacto de sus revistas.

${ }^{* * * * *}$ Pajek es un programa en forma de fichero para representar series temporales y es recomendado para generar mapas de redes sociales.
}

representación de algunas instituciones utilizamos sus siglas por que era como estaban representadas en versión electrónica de la revista investigada.

\section{RESULTADOS Y DISCUSIÓN DE LOS DATOS}

El análisis de este trabajo reunió todos artículos contenidos en las publicaciones de los años de 1995 hasta 2003, donde fueron analizados 278 artículos, de los cuales se tuvieron en cuenta el número de autores:

- 1 autor (total de 185 trabajos),

- 2 autores (52 trabajos),

- 3 autores (22 trabajos),

- más de 3 autores (19 trabajos).

El índice de coautoría estuvo en 1,57, y la representación de artículos por idioma fue de 255 en lengua portuguesa, 19 en lengua española y 4 en lengua inglesa. La investigación realizada estuvo dividida por cada objetivo específico que determinamos en el principio, con un índice de autores más citados; la visibilidad de las citas y sus factores de impacto; los indicadores de las revistas indizadas en Latindex, SciELO (estudiando también sus factores de impacto) y las demás revistas que no están indizadas en ninguna base de datos; las citaciones de libros; de tesis; de actas de congresos; de comunicaciones (documentos internos de las instituciones); de documentos electrónicos; análisis de la relación entre los autores que publicaron dentro de la revista en cuestión; y un análisis de las palabras-clave con las áreas temáticas de las citas utilizadas.

\section{Representación de citas recibidas por autoría}

Para este tipo de análisis, conseguimos recuperar un total de 6.249 citas, donde fueron analizadas, visualizadas y representadas en el grafico 1 .

Los autores representados en este gráfico son los que recibieron 15 o más citas. De todos los autores citados más del 69\% son extranjeros y referencias en el área para Brasil, como Saracevic, Belkin, Lancaster, Wersig, Cronin, Lévy, Porter, Bourdieu, Drucker, Castells, Price, Dervin, Robertson, Foucault, Fox, Haberlas, Meadws y Morin, los demás -autores brasileños- son miembros importantes de las mejores instituciones de investigación del país, como Barreto, Cunha, Freire, Pinheiro, Tarapanoff, Miranda, Araújo y Vieira, todos con una 
GRÁFICO 1

Autores con más de 15 citas

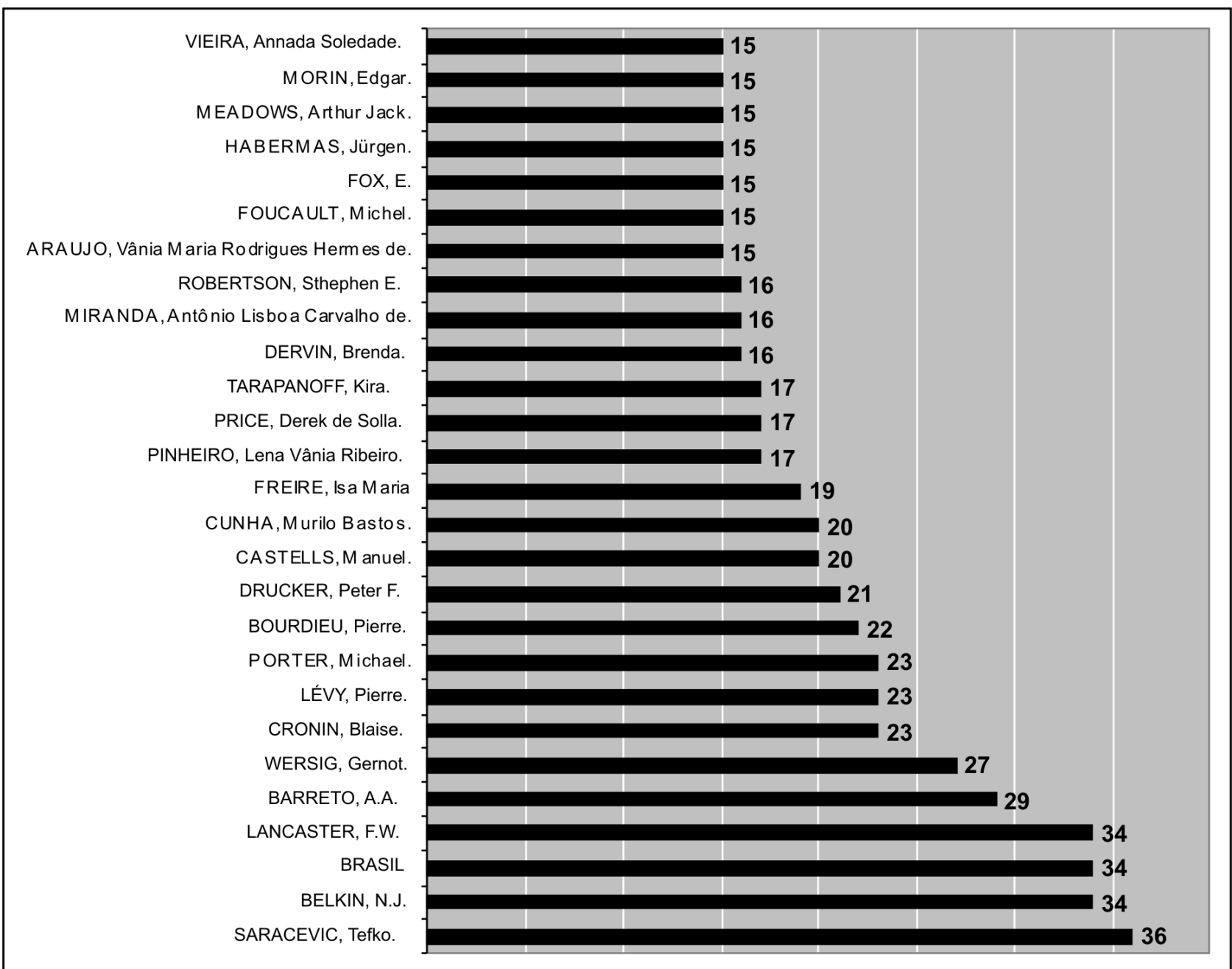

producción científica sólida y muy fuerte en el país, además de que son referencias nacionales para el área de Ciencia y Tecnología brasileña. En relación a gran cantidad de citas recibidas por Brasil (34), es debido a que buena parte de las consultas son de documentos gubernamentales.

En resumen, todos los autores citados con un número superior a 15 citas son investigadores con tradición científica, y trabajan generalmente con sólo una temática. Sin embargo, existen autores de gran importancia en el área de Información y Documentación que tuvieron una cantidad de citas por debajo del análisis, como Ingwersen, Goldmann, Campello, Davenport, Lastres, Montalli, Demo, Figueiredo, Garfield, Velho, Barden, Targino, Schwartzman, Le Coadic y Blattman.
Visibilidad de citas de revistas ISI

A partir de este punto evaluaremos las citas de revistas indizadas en ISI, donde vamos a visualizar la parte cualitativa de los indicadores, y analizar el factor impacto de cada revista (tabla 1$)$.

Del total de citas de revistas indizadas en ISI, fueron recuperados 618 citas, donde fueron representadas por 93 revistas, cuya representación en la tabla fue de 518 citas, identificas con 13 o más citas recibidas

En la parte cuantitativa, la revista más citada fue Journal of American Society for Información Science $\mathcal{E}$ Technology con 89 citas y una regularidad de frecuencia en todos los años, con excepción en los años 2002 y 2003. Las demás revistas tuvieron también una buena citación, como las 57 citas recibidas por la revista Information Processing $\mathbb{E}$ Management, las 47 de Journal of Documentation, y las 43 
TABLA 1

Revistas ISI y su factor de impacto referente a 2002

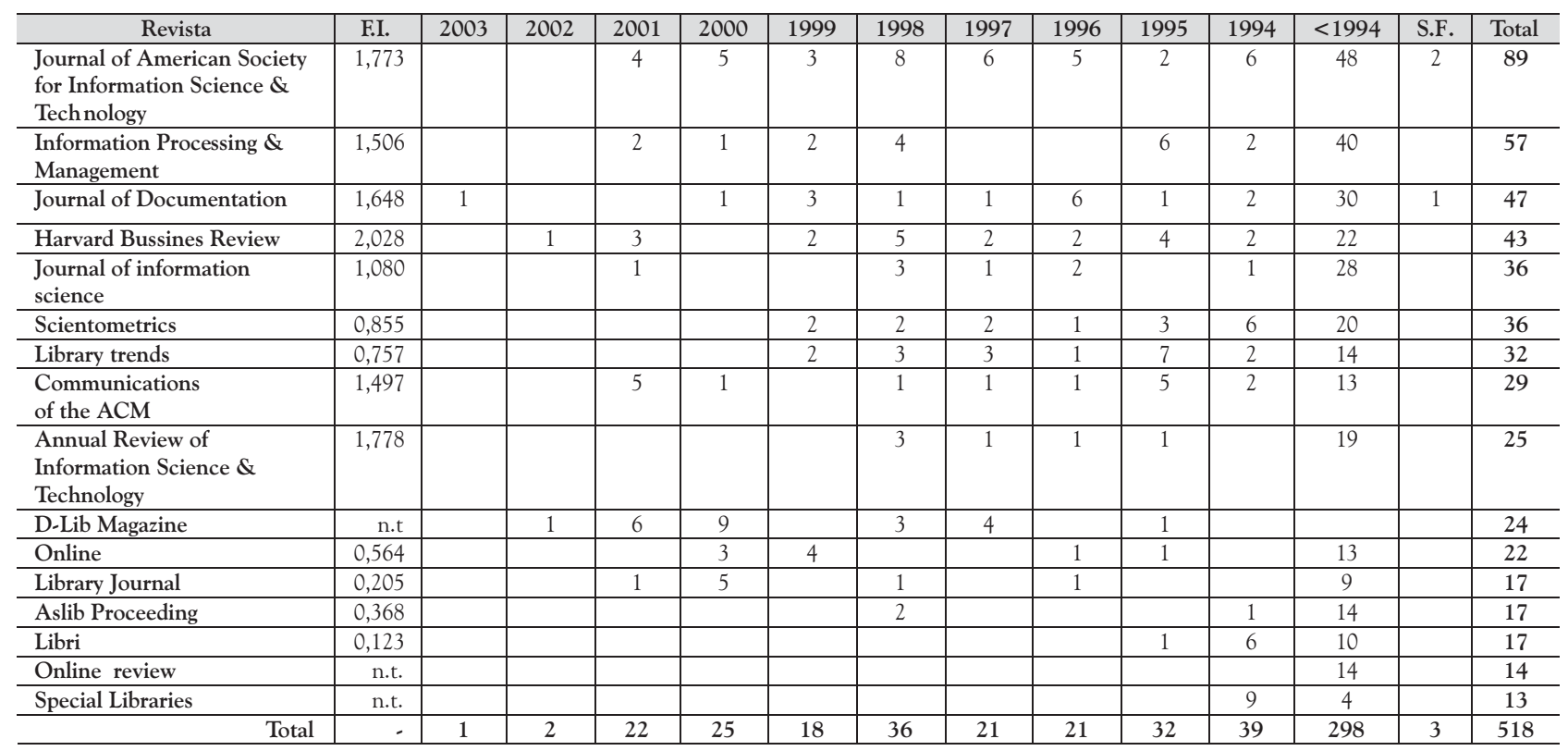

Nota: (n.t. = No tienen factor de impacto / S.F. $=$ Sin fecha).

de Harvard Bussines Review, entre las demás ilustradas en la tabla, demostrando que los autores que publican en la revista Ciência da Informação están consultando fuentes de información muy ricas y de gran calidad, con un porcentaje muy representativo, que son las revistas indizadas en ISI.

En el análisis de los datos entre los años con mayor representabilidad, observamos que 1994, 1995 y 1998 tuvieron una frecuencia por encima de 30 citas, seguido de los años de 1996, 1997, 2000 y 2001 que tuvieron un índice por encima de 20 citas. Sin embargo, los años de 2003 y 2002 tuvieron el menor índice, tan sólo dos citas, con representación de las revistas Journal of Documentation y Harvard Bussines Review.

El factor de impacto aquí representado parece un poco distante de la realidad de frecuencia de las revistas citadas, donde algunas poseen un factor muy elevado debido su publicación mensual y otras con un índice bajo debido a que su edición es trimestral, cuatrimestral y semestral.

La revista con mayor factor de impacto fue Harvard Bussines Review con un índice de 2.028, hecho que se debe principalmente a su publicación mensual, seguida de la revista Annual Review of Information Science 8 Technology (1.778), y de la revista Journal of American Society for Información Science $\mathcal{E}$ Technology (1.773) que publican un número muy elevado de artículos por año en sus ediciones. La secuencia es representada por la revista Journal of Documentation, Information Processing $\mathbb{E}$ Management, Communication of ACM y Journal of Information Science con un índice de factor de impacto superior a 1. Las demás revistas que poseen un índice menor a 1 son revistas de publicación trimestral y cuatrimestral, algunas con poca representatividad dentro del área de Información y Documentación, alejado de los enfoques principales de los autores que publican dentro de la revistas Ciência da Informação, a excepción de las revistas Scientometrics y Library Journal, que son vitales para el área de información.

Visibilidad de las revistas indizadas en Latindex, SciELO y otras revistas no indizadas en ninguna base de datos

Otra cuestión representada en este trabajo, es sobre las revistas que no están indizadas en ISI, a las que nos referimos en muchas ocasiones como revistas indizadas en Latindex, SciELO y otras fuentes.

Estas revistas tienen la misma importancia que las revistas indizadas en ISI, sin embargo, dividimos y denominamos como no ISI para tener un parámetro de trabajo.

En este análisis identificamos las revistas más citadas y también el factor de impacto de revistas que están indizadas en SciELO, como podemos visualizar en la tabla 2. 
TABLA 2

Revistas citadas indizadas en Latindex, SciELO y otras que no están presentes en ninguna base de datos

\begin{tabular}{|c|c|c|c|c|c|c|c|c|c|c|c|c|c|c|}
\hline Revista & F.I. & 2003 & 2002 & 2001 & 2000 & 1999 & 1998 & 1997 & 1996 & 1995 & 1994 & $<1994$ & s.f. & Total \\
\hline Ciência da Informação & 0,2200 & 2 & 3 & 11 & 28 & 16 & 42 & 26 & 29 & 35 & 16 & 134 & 1 & 343 \\
\hline $\begin{array}{l}\text { Revista de Biblioteconomia } \\
\text { de Brasilia }\end{array}$ & n.t. & & & & & & 1 & & 2 & 1 & & 26 & 1 & 31 \\
\hline Transinformaçao & n.t. & & & & & 5 & & 14 & 4 & 2 & 1 & 4 & & 30 \\
\hline $\begin{array}{l}\text { Revista da Escola de } \\
\text { Biblioteconoma da UFMG }\end{array}$ & n.t. & & & & & & & & & & 6 & 22 & & 28 \\
\hline $\begin{array}{l}\text { Perspectivas em Ciência da } \\
\text { Informação }\end{array}$ & n.t. & & & & 1 & 2 & 2 & 5 & 14 & & & & & 24 \\
\hline Eletronic Library & n.t. & & & & & & 1 & & & 3 & 7 & 9 & & 20 \\
\hline Informare & n.t. & & & & & & & 9 & 3 & 7 & & & & 19 \\
\hline Ciência e Cultura & n.t. & & & & & & & & & & & 15 & & 15 \\
\hline Folha de S. Paulo & n.t. & & 3 & & 1 & 4 & 2 & 1 & & 1 & 1 & 1 & & 14 \\
\hline $\begin{array}{l}\text { Information Technologies } \\
\text { and Libraries }\end{array}$ & n.t. & & & & & & & & & 4 & 5 & 4 & & 13 \\
\hline Sao Paulo em Perspectiva & 0,0609 & & 1 & & & & 3 & & & & 9 & & & 13 \\
\hline $\begin{array}{l}\text { Documentaliste - Sciences } \\
\text { de l'Information }\end{array}$ & n.t. & & & & & 1 & & & 1 & 3 & 4 & 2 & & 11 \\
\hline $\begin{array}{l}\text { Revista de Administraçao } \\
\text { de Empresa }\end{array}$ & n.t. & & & & & & 2 & & & & 1 & 8 & & 11 \\
\hline $\begin{array}{l}\text { College \& Research } \\
\text { Libraries }\end{array}$ & n.t. & & & & & 1 & 2 & & 1 & & 2 & 4 & & 10 \\
\hline $\begin{array}{l}\text { Revista Brasileira de } \\
\text { Biblioteconomia e } \\
\text { Documentação }\end{array}$ & n.t. & & & & & 1 & & & & & & 9 & & 10 \\
\hline $\begin{array}{l}\text { Bulettin des Bibliothèques } \\
\text { de France }\end{array}$ & n.t. & & & & & & & & 3 & 1 & 2 & 4 & & 10 \\
\hline Total & - & 2 & 7 & 11 & 30 & 30 & 55 & 55 & 57 & 57 & 54 & 233 & 2 & 602 \\
\hline
\end{tabular}

Nota: (n.t. = No tiene factor de impacto).

La cantidad de citas fueron de 2.023 indizadas en 492 revistas, y están representados sólo un 29,75\%, que son las revistas que obtuvieron un número de citas superior o igual a 10.

En el análisis quedó evidente que existe una gran cantidad de autocitas de la revista Ciência da Informação, pues esta revista es referencia nacional en Ciencia y Tecnología, siendo también considerada por muchos investigadores de América Latina como la más importante del área en Brasil.

Las representación de frecuencias de las revistas citadas son Ciência da Informação con la mayor denominación numérica (343), seguida de la Revista de Biblioteconomia de Brasilia, Transinformação, Revista da Escola de Biblioteconomia da UFMG, Perspectivas em Ciência da Informação, y Eletronic Library con un índice superior a 20 citas, las demás revistas en la tabla 2 obtuvieron una frecuencia por encima de 10 citas recibidas.

Es importante mencionar que la Revista da Escola de Biblioteconomia da UFMG pasa a ser denominada como Perspectivas em Ciência da Informação a partir de 1996, cuya sumatoria final de esta revista es de 52 citas recibidas, siendo por lo tanto a segunda mayor fuente de consumo nacional por parte de los autores.

Dentro de estas revistas, algunas son parte del sistema Latindex, como Ciência da Informação, Ciência e Cultura, y São Paulo em Perspectiva. Sin embargo, existen algunas revistas brasileñas que están en la tabla y que no forman parte de este sistema (Latindex), aún así son reconocidas y recomendadas en Brasil, como la Revista de Biblioteconomia de Brasilia, Revista de Administração de Empresa y la Perspectivas em Ciência da Informação (esta última hace parte del paquete Francis del Instituto de Información Científica y Tecnológica de Francia) y las revistas que hacen parte de la base de datos SciELO, que son representadas por la revista Ciência da Informação y São Paulo em Perspectiva y que también son visualizadas con un índice de factor de impacto en la tabla 2,

Sus factores de impacto son de poca representabilidad ante los visualizados en las revistas ISI, sin embargo, son iniciativas recientes de la base de datos y que merecen ser valoradas, aunque es sabida la dificultad para mantener la edición puntual de revistas brasileñas, debido la falta de incentivos y de trabajos que no están a la altura de la calidad de algunas revistas. 
En este tipo de revista, los años que tuvieron una mayor frecuencia fueron de 1994 hasta 1998, con un índice por encima de 50 citas recibidas, seguida de los años 1999 y 2000 con una frecuencia superior a 30 citas. Los demás años (2001 hasta 2003) no recibieron muchas citas, por ser años muy próximos al límite del periodo estudiado.

\section{Citaciones de Libros}

La relación de citas de libros fue muy inusual, auque se refleja un cambio radical de los datos analizados hasta este momento, donde son representados por muchos países con un índice muy elevado, próximo al análisis de citas de revistas. Su influencia para la publicación fue muy elevada, por la buena cantidad de citas utilizadas en lengua portuguesa, contrastando con el elevado índice de trabajos firmados en lengua portuguesa, donde la cifra es de 1.120 citas entre Brasil y Portugal, seguido por la citas de lengua inglesa con un total de 697 citas (Estados Unidos; Reino Unido; Canadá y Holanda), y las citas de lengua española, identificadas con 60 citas divididas por España, México y Venezuela. Las citas representadas en lengua francesa fueron sólo representadas por Francia, con un total de 153, como sigue su visualización en la tabla 3.

Los años que tuvieron un mayor índice de citas en el periodo analizado, fueron los comprendidos entre 1994 y 1998. En relación al contexto individual de los países hubo una oscilación en 1994, 1996 y 1997, donde cada país destacó de forma particular.

Por otro lado, el campo destinado a los años anteriores a 1994, tuvo un índice muy elevado, pero este campo aquí empleado sufre la misma relación que se dio en la tabla 1, un período muy elevado. Sin embargo, muchas de las citas consultadas no fueron representadas por país y tampoco por año de publicación, por lo que convenimos que fueron citas sin fecha y sin hogar, donde su visualización estuvo en 12 trabajos. Hubo muchos trabajos que identificamos el año de publicación pero no conseguimos identificar su país de origen, como es representado en la tabla 3.

\section{Citaciones de Tesis}

Otra análisis que procuramos identificar fueron las citas a tesis, pues esta tipología tiene una representación muy curiosa. Este tipo de recurso no es muy utilizado por los investigadores, debido al acceso a este tipo de cita y por qué existe una relación muy fuerte de los derechos de autoría, que es controlado por las instituciones que poseen los ejemplares.

La discusión para este tipo de citas fue llevada a cabo con una metodología similar al análisis realizado en las citas a libros, donde estudiamos los países, con intención de observar qué idioma han utilizado los autores que publican dentro de la revista, y también, un análisis representativo por año.

Este tipo de análisis no obtuvo un índice elevado en un determinado año, la oscilación se da entre 5 y 15 citas, manteniendo una constante entre esas cifras a excepción del período anterior a 1994, donde fue muy citado, con una influencia muy elevada de citas de las décadas de 1970 y 1980 , con un $83,5 \%$ de todas las citas utilizadas (no representada en la tabla debido al espacio físico).

TABLA 3

Visualización de los libros citados por año y por países representados

\begin{tabular}{|c|c|c|c|c|c|c|c|c|c|c|c|c|c|}
\hline País & 2003 & 2002 & 2001 & 2000 & 1999 & 1998 & 1997 & 1996 & 1995 & 1994 & $<1994$ & S.F. & Total \\
\hline Brasil & 1 & 16 & 25 & 50 & 66 & 64 & 92 & 70 & 66 & 101 & 518 & 3 & 1.072 \\
\hline EE UU & & 1 & 6 & 11 & 14 & 27 & 25 & 15 & 14 & 18 & 237 & 2 & 370 \\
\hline Francia & & & 3 & 2 & 3 & 2 & 6 & 15 & 14 & 9 & 99 & & 153 \\
\hline S.H. & & 2 & & 2 & & 7 & 3 & 6 & 5 & 1 & 11 & 12 & 49 \\
\hline Portugal & & & & & 2 & & 4 & 2 & & 5 & 32 & 3 & 48 \\
\hline Canadá & & & & 1 & & & 1 & & 2 & 2 & 18 & & 24 \\
\hline Holanda & & & 1 & & & & & & & 1 & 14 & & 16 \\
\hline México & & & & & & & 1 & 1 & 2 & 1 & 10 & & 15 \\
\hline Venezuela & & & & & & & & 4 & & & 6 & & 10 \\
\hline Total & 1 & 19 & 42 & 72 & 94 & 114 & 138 & 136 & 115 & 155 & 1.172 & & 2.079 \\
\hline
\end{tabular}

Nota: S.F. = Sin Fecha; y S.H. Sin Hogar 
TABLA 4

Tesis citadas en el período de 1995 a 2003 según los países

\begin{tabular}{l|c|c|c|c|c|c|c|c|c|c|c|c}
\hline \multicolumn{1}{c|}{ Países } & 2003 & 2002 & 2001 & 2000 & 1999 & 1998 & 1997 & 1996 & 1995 & 1994 & $<1994$ & Total \\
\hline Brasil & 3 & 6 & 9 & 10 & 12 & 14 & 7 & 9 & 6 & 14 & 73 & 163 \\
\hline España & & 1 & & & & & & & & & 16 & 17 \\
\hline U.K. & & & & & & & & & & & 11 & 11 \\
\hline Francia & & & & & & & 1 & & 2 & & 2 & 5 \\
\hline Portugal & & & & & & & & & & 1 & 1 & 2 \\
\hline $\begin{array}{l}\text { Costa } \\
\text { Rica }\end{array}$ & & & & & & & & & 1 & & & 1 \\
\hline EE UU & & & & & & & & & & & 1 & 1 \\
\hline Suiza & & & & & & & & & 1 & & & 1 \\
\hline Total & 3 & 7 & 9 & 10 & 12 & 14 & 8 & 9 & 10 & 15 & 104 & 201 \\
\hline
\end{tabular}

La representación por idiomas utilizados (país), indica que una vez más la lengua portuguesa fue más representada con un total de 165 citas entre Brasil y Portugal, seguido por la lengua española con 18 citas (España y Costa Rica), la lengua inglesa con 13 citas (Reino Unido, Suiza y Estados Unidos), seguido por citas de lengua francesa con 5 citas (Francia). En esto análisis la lengua tiene una influencia fundamental.

\section{Presentaciones de actas de congresos citadas}

Otro tipo de cita contemplada es la de trabajos presentados en congresos, coloquios, jornadas y encuentros.

Este recurso es muy interesante, porque generalmente muchos de los trabajos presentados en esto tipo de encuentros son futuros artículos de revistas, con modificaciones del modelo original debido a cambio de información y de pistas que otros participantes acaban sugiriendo.

Durante el período de 2003-2002 hubo una citación baja en esta tipología de cita, concentrándose la gran mayoría en los años anteriores a 1994, donde la investigación mostró que en la década de 1970 se realizaron un gran número de citas, principalmente ocurridos en India debido al gran desarrollo de software en el universo de la información y de la teoría aplicada por Ranganathan.

La secuencia de la representación por años destaca 1994 y 1995 con una media superior a las 54 citas recibidas. Otro período en la escala fueron de 1996 hasta 2001 donde la media de citas utilizadas fue entre 20 y 33, demostrando una constancia de las citas más recientes por parte de los autores que publicaron en la revista Ciência da Informação.

TABLA 5

Citas de trabajos presentados en actas de congresos según los países

\begin{tabular}{l|c|c|c|c|c|c|c|c|c|c|c|c|c}
\hline País & 2003 & 2002 & 2001 & 2000 & 1999 & 1998 & 1997 & 1996 & 1995 & 1994 & $<1994$ & S.F. & Total \\
\hline Brasil & - & 1 & 8 & 11 & 3 & 12 & 16 & 15 & 6 & 35 & 47 & - & 154 \\
\hline EE UU & 3 & 3 & 10 & 6 & 7 & 8 & 9 & 6 & 6 & 3 & 22 & - & 83 \\
\hline India & - & - & - & - & 1 & - & - & - & 24 & 14 & 39 & - & 78 \\
\hline S.H. & - & - & - & - & - & - & - & - & - & - & - & 33 & 33 \\
\hline UK & - & 1 & 1 & - & 2 & 3 & 3 & 1 & 7 & - & 6 & - & 24 \\
\hline Canadá & - & - & 3 & - & 4 & 3 & - & 1 & 4 & 1 & 4 & - & 20 \\
\hline Alemania & - & - & 2 & 1 & 1 & 2 & - & - & 1 & 3 & 7 & - & 17 \\
\hline Francia & - & - & 2 & 1 & - & - & - & 1 & 1 & 2 & 9 & - & 16 \\
\hline España & - & - & 3 & 3 & - & 2 & - & - & - & 1 & 4 & - & 13 \\
\hline Finlandia & - & - & - & - & - & - & - & - & 2 & 3 & 4 & - & 9 \\
\hline Holanda & - & - & - & 1 & - & 2 & - & 1 & 2 & - & 3 & - & 9 \\
\hline Cuba & - & 1 & - & - & 2 & 1 & 1 & 2 & 1 & 1 & - & - & 9 \\
\hline Total & 3 & 6 & 29 & 23 & 20 & 33 & 29 & 27 & 54 & 63 & 145 & 33 & 465 \\
\hline
\end{tabular}

Nota: S.H. = Sin Hogar; y S.F. = Sin Fecha 
Es importante mencionar el gran número de citas que no fueron identificadas las direcciones y las fechas del documento, donde se recuperaron 33 citas. Concluimos que no hubo una normalización adecuada para estas citaciones.

En relación a las citas por idiomas, la mayor representación fue de la lengua inglesa con un total 240 citas (representado por Estados Unidos, India, Reino Unido, Canadá, Alemania, Finlandia y Holanda), seguido por la lengua inglesa portuguesa con 154 citas (representado por Brasil), seguido por citas de lengua española con 22 (representados por España y Cuba) y por las citas en lengua francesa con 16 citas (visualizada por Francia). Un detalle muy interesante, es que las citas representadas por Canadá son todas en lengua inglesa.

Citas por documentos internos de las instituciones (representados como comunicaciones)

Pasamos ahora a analizar las citas en forma de comunicaciones. Este tipo de material generalmente es constituido por los departamentos y órganos gubernamentales de los países, como normas, comunicaciones entre instituciones, directrices e informes.

Este recurso fue poco citado por los autores que publicaron en la revista Ciência da Informação, sin embargo su representación quedó evidente en los siguientes idiomas: lengua portuguesa (138 citas - Brasil y Portugal); seguido por la lengua inglesa (45 citas - Estados Unidos, Suiza, Reino Unido y Canadá con 3 de las 5 citas), en la secuencia con mayor representabilidad fue la lengua española con 24 citas (representada por España y Uruguay), y seguida por la lengua francesa con 13 citas (representada por Francia y Canadá con 2 citas de 5 recibidas).
Los años que tuvieron un mayor índice de citas en el periodo fueron las fechas anteriores a 1994 (con un periodo muy extenso), 1994, 1995, 1996, 1997, 1999, 2000 y 2001 con una citación por encima de 15 consultas. Para los demás años $(1998,2002$ y 2003) la media estuvo entre las 2 hasta 12 citas, teniendo una gran variación de año en año, como puede ser visualizada en tabla 6.

\section{Citas de documentos electrónicos}

Para las citas electrónicas, esta tipología fue representada por correo electrónico, URL e informaciones de bases de datos, conforme se visualiza en la tabla 7 .

Este recurso fue poco citado por los autores que publicaron en la revista Ciência da Informação, sin embargo su representación quedó evidente en los siguientes idiomas: lengua portuguesa (138 citas - Brasil y Portugal); seguido por la lengua inglesa (45 citas - Estados Unidos, Suiza, Reino Unido y Canadá con 3 de las 5 citas), en la secuencia con mayor representabilidad fue la lengua española con 24 citas (representada por España y Uruguay), y seguida por la lengua francesa con 13 citas (representada por Francia y Canadá con 2 citas de 5 recibidas).

Los años que tuvieron un mayor índice de citas en el periodo fueron las fechas anteriores a 1994 (con un periodo muy extenso), 1994, 1995, 1996, 1997, 1999, 2000 y 2001 con una citación por encima de 15 consultas. Para los demás años $(1998,2002$ y 2003) la media estuvo entre las 2 hasta 12 citas, teniendo una gran variación de año en año, como puede ser visualizada en tabla 6.

\section{Citas de documentos electrónicos}

Para las citas electrónicas, esta tipología fue representada por correo electrónico, URL e informaciones de bases de datos, conforme se visualiza en la tabla 7.

\section{TABLA 6}

Citas de Comunicaciones por año y por países

\begin{tabular}{l|c|c|c|c|c|c|c|c|c|c|c|c}
\hline País & $\mathbf{2 0 0 3}$ & $\mathbf{2 0 0 2}$ & $\mathbf{2 0 0 1}$ & $\mathbf{2 0 0 0}$ & $\mathbf{1 9 9 9}$ & $\mathbf{1 9 9 8}$ & $\mathbf{1 9 9 7}$ & $\mathbf{1 9 9 6}$ & $\mathbf{1 9 9 5}$ & $\mathbf{1 9 9 4}$ & $<1994$ & Total \\
\hline Brasil & 2 & 8 & 9 & 13 & 17 & 5 & 8 & 8 & 12 & 13 & 35 & 130 \\
\hline EE UU & & & & 3 & & 4 & 4 & 3 & 2 & 2 & 9 & 27 \\
\hline España & & 1 & 1 & 6 & 1 & & 1 & 3 & 1 & 1 & 4 & 19 \\
\hline Francia & & 1 & & 2 & 3 & 1 & & 1 & & & 3 & 11 \\
\hline Suiza & & & & 1 & & 2 & & 2 & & 1 & 2 & 8 \\
\hline Portugal & & & 2 & 1 & & & 2 & 3 & & & & 8 \\
\hline UK & & & 1 & & & & 2 & 2 & 1 & & 1 & 7 \\
\hline Uruguay & & & 2 & & & & 1 & & & 1 & 1 & 5 \\
\hline Canadá & & & 1 & & & & & & 1 & 1 & 2 & 5 \\
\hline Total & 2 & 10 & 16 & 26 & 21 & 12 & 16 & 22 & 17 & 19 & 57 & 220 \\
\hline
\end{tabular}


TABLA 7

Citas de materiales electrónicos por año y por países

\begin{tabular}{|l|c|c|c|c|c|c|c|c|c|c|c|c|c|}
\hline Países & 2003 & 2002 & 2001 & 2000 & 1999 & 1998 & 1997 & 1996 & 1995 & 1994 & $<1994$ & S.F. & Total \\
\hline S.H. & & & & & & & & & & & & 143 & 143 \\
\hline Brasil & 2 & & 1 & 4 & 3 & 8 & 2 & 3 & & & 3 & 60 & 86 \\
\hline EE UU & & & 2 & 10 & 7 & 6 & 6 & 7 & 5 & 4 & 8 & 26 & 81 \\
\hline UK & & & & & 4 & 2 & 1 & 2 & 2 & 2 & 4 & 16 & 33 \\
\hline Francia & & & & 2 & 2 & 1 & 3 & & & & & 5 & 13 \\
\hline Canadá & & & & 1 & 1 & 3 & 3 & 1 & & & & 1 & 10 \\
\hline España & 1 & & & 4 & 1 & & & & & & & 3 & 9 \\
\hline Alemania & & & & & 4 & 1 & & & & & & 4 & 9 \\
\hline Total & 3 & - & 3 & 21 & 22 & 21 & 15 & 13 & 7 & 6 & 15 & 258 & 384 \\
\hline
\end{tabular}

Nota: S.H. = Sin Hogar; y S.F. $=$ Sin Fecha

La mayor representación visible en esta tabla son las fuentes que no pueden ser localizadas por el país (en general en lengua inglesa, portuguesa y una buena representación sin hogar) y también el año (sin fecha), ya que este tipo de cita generalmente no siguió un patrón como las demás tipologías.

Los idiomas más utilizados son inglés (130 citas entre Estados Unidos, Reino Unido, Alemania y Canadá con 7 de 10 recibidas) portugués (86 citas de Brasil), francés (19 citas de Francia y Canadá con 3 de 10 recibidas) y español (9 citas de España). Sin embargo, tuvo muchas citas recibidas de las que no fue posible identificar el país y tampoco el año; de las 143 citas 79 estaban en lengua portuguesa y 64 en lengua inglesa.

Los años de 1994, 1995, 2001 y 2003 tuvieron un índice entre 3 y 13 citas, mientras que los años de 1997 hasta 2000 tuvieron el mayor índice de citaciones, con una media entre 15 y 22. El año de 2002 no tuvo ninguna cita representada.

Análisis de la relación entre los autores de los artículos de la revista Ciência da Informação

Otro aspecto estudiado fueron las relaciones de los autores que publicaron en la revista (redes sociales). Esas relaciones se resumen en forma de nodos y enlaces como observamos en el Gráfico 2, dibujando un mapa conceptual en relación a las colaboraciones de los autores.

\section{GRÁFICO 2} hasta 2003
Si atendemos a las firmas de los artículos, se pueden identificar las colaboraciones institucionales dentro de la revista Ciência da Informação, donde destacamos la Universidad de Sao Paulo (USP) y sus colaboradores (Pontificia Universidad Católica de Sao Paulo; SciELO; Conjunto de Química; Universidad Federal de Espirito Santo; Universidad Autónoma de Madrid, Pontificia Universidad Católica del Paraná; Universidad Federal del Río Grande del Sur; Universidad Federal del Río Grande del Norte; Universidad Federal del Rio de Janeiro y demás unidades de la Universidad de Sao Paulo), como la principal red del análisis.

Relaciones de las instituciones que publican en la revista en el periodo de 1995

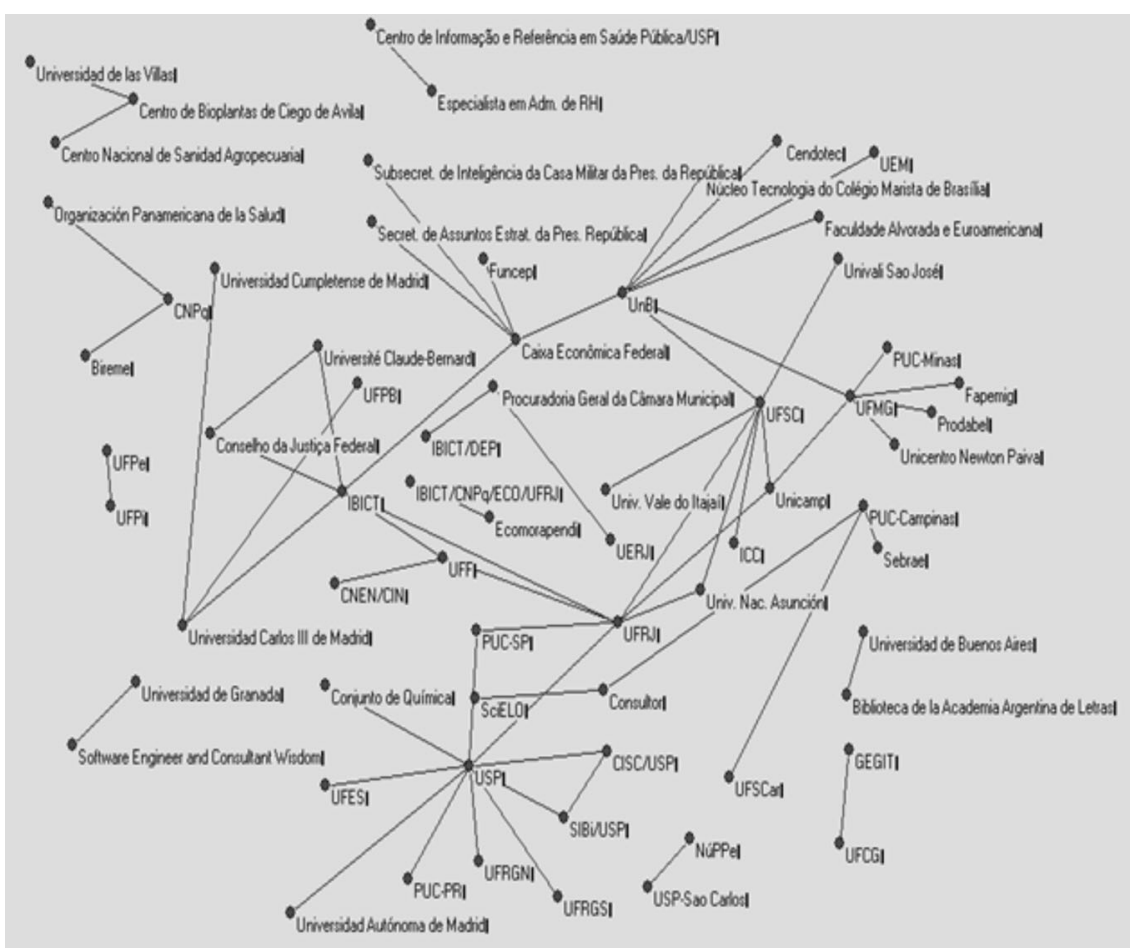


Otra institución que tuvo un índice elevado fue la Universidad de Brasilia (con colaboración con el Cendotec; Caja Económica Federal; Universidad Federal de Santa Catarina; Universidad Estadual de Maringá; Núcleo de Tecnología del Colegio Marista; Facultad Alvorada y Euroamericana y la Universidad Federal de Minas Gerais). Lo mismo ocurrió con la Universidad Federal de Santa Catarina (Universidad de Brasília; Univali San José; Unicamp; ICC; Universidad Nacional de Asunción; Universidad Federal de Río de Janeiro y Universidad Vale del Itajaí) y con la Universidad Federal de Minas Gerais (colaboración con Universidad de Brasilia; Pontificia Universidad Católica de Minas Gerais; Fapemig; Prodatel y Unicentro Newton Paiva).

La Universidad Federal do Rio de Janeiro (UFRJ) mantuvo su colaboración con Ibict; Universidad Nacional de Asunción; Universidad de Sao Paulo; Unicamp; Universidad Federal de Santa Catarina; Universidad Federal Fluminense y Pontificia Universidad Católica de Sao Paulo. Sin embargo, es fundamental mencionar que la UFRJ estuvo vinculada con el Ibicit hasta 2003, con el programa de Postgrado, pero nosotros identificamos el origen de los autores en los artículos conforme a entrada de datos de los mismos.

El Ibict, órgano importante en el ámbito científico nacional y responsable por la edición de la revista tuvo su programa de Postgrado representado por la UFRJ, como ha sido mencionado anteriormente, pero desde muchos años que su relación con la Universidad Federal Fluminense es intensa, y esto se concluyó a partir de 2004, con la consolidación del Programa de Postgrado del Ibict en la UFF.

Dentro del universo científico del Ibict, destacamos otras relaciones de gran visibilidad, como el Consejo de Justicia Federal; la Caja Económica Federal; la Universidad Carlos III de Madrid y la Université Claude-Bernarad.

Otro aspecto importante en el análisis de las colaboraciones científicas fue la baja relación dentro de la revista de instituciones de gran prestigio nacional, como de la Universidad Estadual de Campinas, la Bireme y la Pontificia Universidad Católica de Campinas.

\section{Análisis de las palabras-clave con las área temáticas de} las citas

Finalmente, como último análisis visualizamos la relación entre las citas utilizadas por los autores con las palabras-clave indicadas en sus trabajos bibliográficos (artículos en la revista Ciência da Informação). De todo el universo investigado (230 términos), solamente algunas palabras no tuvieron relación alguna con las revistas descritas en las citas, debido a una generalidad de los términos utilizados por los autores, como: Agenda 21, Agroindustria de alimentos, Análisis funcional, Autor permanente, Autor transiente, Censura, Decifración, Derecho social, Derechos autoriales, Ecología, Economía de la lengua, Entropía, Propiedad intelectual, Self-service.

Otra observación que hemos realizado es que muchas revistas no discriminan ningún área temática, siendo denominada siempre como multidisciplinar, como ocurrió en las revistas que están indizadas en ISI (WIRED; Daedalus; Jama; Méta; Minerva; RQ; Federation Proceedin; Science; Nature), para las demás revistas que no están indizadas en ISI ocurrió lo mismo, donde investigamos en sus respectivas páginas web (Revue Tires-Monde; Serial Review; Atlas Bulletin; Mecjournal; Working Paper CBS; Program; Sigir; Fase), en algunos casos muchas de las citas eran de periódicos de edición diaria (Folha de S. Paulo; $\mathrm{O}$ Estado de S. Paulo; O Globo;) y otras publicaciones sin representación científica, como la revista Veja e Istoé.

En relación a los demás tipos de citas consultadas, utilizamos como factor de evaluación los títulos de las publicaciones (libros, actas de congreso, comunicados, documentos electrónicos), siempre utilizando el sentido común y en algunos casos buscando en la web datos sobre el documento citado.

\section{CONCLUSIONES}

Este trabajo priorizó hacer un estudio del análisis de citaciones de los materiales bibliográficos utilizados por los autores que publicaron en la revista Ciência da Informação, por lo que concluimos que:

- La visibilidad más determinante en este trabajo fueron las citas de las revistas ISI, principalmente por el elemento del factor de impacto que representó la calidad de las citas utilizadas. En relación al comportamiento por años, el periodo mas citado fue en 1995 y 1998, denominados como los picos de crecimiento de fuentes utilizadas, sin embargo los demás años también tuvieron un índice respetable, principalmente por los años de 1999 hasta 2003, debido la cuestión de fuentes actuales. Y las revistas más citadas, con una puntuación por en cima de 30 trabajos citados fueron: JASIS (89 citas), Information Processing $\mathcal{E}$ Management, Journal of Documentation, Harvard Bussines Review, Journal of Information Science y Scentometrics, que son referencias obligatorias para cualquier investigador; 
- Las revistas (no indizadas en ninguna base de datos o indizadas en bases de datos Latinoamericanas "Latindex y SciELO”) tuvieron un periodo constante entre 1994 y 1998, sin embargo, de 1999 hasta 2001 fue el periodo con menor número de citas recibidas, principalmente porque son documentos muy recientes y todavía no muy consultados;

- Las revistas con mayores citas fueron Ciência da Informação, Revista de Biblioteconomia de Brasília y Transinformação con por lo menos 30 citas recibidas. Es fundamental mencionar que en casi todas las evaluaciones de citas por revistas científicas la mayoría de las citas son para está revista, lo mismo ocurrió en esto análisis, donde la revista Ciência da Informação obtuvo un porcentual de 12,98 de todas las citas recibidas en forma de revista, justificando la importancia de este medio de comunicación en Brasil y en América Latina;

- Los autores más citados son Saracevic, Belkin, Lancaster, Barreto, Wersig, Cronin, Lévy, Porter, Bourdieu, Drucker, Castells, Cunha, Freire, Pinheiro, Price, Tarapanoff, Dervin, Miranda, Robertson, Araújo, Foucault, Fox, Haberlas, Meadows, Morin y Vieira, donde algunos son clásico internacionales y otros exponentes de la Ciencia de la Información y de la Ciencia y Tecnología brasileña;

- En relación a los libros consultados por los autores, se constató que los años de mayor número de citas fueron 1994 y 2000. Los años de 1996 y 1997 tuvieron un porcentual muy positivo, siendo la gran mayoría de la literatura portuguesa (obras utilizadas de Brasil y Portugal), seguida de la inglesa (obras utilizados de EE UU; Reino Unidos y Canadá) y la francesa (obras de Francia y Canadá);

- Las citas de tesis utilizadas por los autores tuvieron un índice bajo, con una relativa representación en los años de 1994 y 1998. Esta tipología podría y debería ser más consultada debido la gran concentración de informaciones innovadoras que contienen, pero también sabemos que este tipo de material editorial no es de fácil acceso, dificultando casi siempre su consulta por los investigadores;

- Las demás tipologías estudiadas (Trabajos presentados en Actas de Congresos; Comunicaciones y Materiales Electrónicos) tuvieron una mayor representación en la literatura de lengua portuguesa, representadas principalmente por Brasil y por ser la lengua vernácula de buena parte de los autores.
- Las actas de congresos tuvieron mejor representación en los años de 1994 y 1995, con la particularidad que India fue representado por primera vez y con un porcentaje muy significativo;

- Los materiales en forma de comunicaciones tuvieron una mejor utilización en los años de 1996, 1999 y 2000;

- Y los materiales electrónicos fueron utilizados con mayor intensidad en 1998 y 2000, teniendo también una gran representación de fuentes que no contenían año y lugar;

- Las relaciones en forma de colaboraciones institucionales en las publicaciones de los artículos tuvieron pocos nodos centrales, representados por Universidad de Sao Paulo; Universidad Federal de Santa Catarina; Universidad de Brasilia; Universidad Federal de Minas Gerais y el propio Ibict (órgano responsable por la edición de la revista investigada). Las demás colaboraciones no tuvieron una relación por encima de tres instituciones participantes;

Finalmente, para la relación de las palabras-clave con la temática de las citas utilizadas pocas fuentes no fueron identificadas, con principal destaque para algunos periódicos no científicos, como Folha de S. Paulo, O Estado de S. Paulo, O Globo, Veja e Istoé.

Artigo submetido em 01/06/2006 e aceito em 07/03/2007.

\section{REFERENCIAS}

BIOJONE, Maria Rocha. Forma e função dos periódicos científicos na comunicação da ciência. 2001. 107 f. Tese (Mestrado em Ciência da Informação)- Escola de Comunicação e Artes, Universidade de São Paulo, São Paulo, 2001.

BRADFORD, Samuel Clemente. Documentation. Londres: Crosby Lockwood and Son, 1948.

FAZITO, Dimitri. A análise de redes sociais (ARS) e a migração: mito e realidade. In: ENCONTRO DA ASSOCIAÇÃO BRASILEIRA DE ESTUdOS POPUlACIONAIS, 13., 2002, Ouro Preto. Anais eletrônicos... Disponível em: <http://www.abep.nepo.unicamp.br/docs/ anais/pdf/2002/GT_MIG_ST1_Fazito_texto.pdf>. Acesso em: 23 maio 2005.

FERREIRA, Maria Cecília Gonzaga. Avaliação de periódicos científicos. Ceará: UFC, 2001. Disponível em: <http://www.biblioteca.ufc.br/ cecilia.ppt>. Acesso em: 30 jun. 2003.

GARFIELD, Eugene. Citation analysis as a toll in journal evaluation. Science, Washington, v. 178, n. 4060, 1972.

. Fortnightly review: how can impact factors be improved? British Medical Journal, London, n. 3, p. 313-411, 1996. 
The impact factor. Current Contents, Philadelphia, v. 20, n. 25, p. 3-8, June 1994.

MACIAS-CHAPULA, César A. O papel da informetria e da cienciometria e sua perspectiva nacional e internacional. Ciência da Informação, Brasília, v. 27, n. 2, p. 134-140, 1998.

MOYA-ANEGÓN, Félix et al. Atlas de la ciencia española: propuesta de un sistema de información científica. Revista Española de Documentación Científica, Madrid, v. 27, n. 1, p. 11-29, 2004.

MUELLER, Suzana Pinheiro Machado; PECEGUEIRO, Claúdia Maria Pinho de Abreu. O periódico Ciência da Informação na década de 90: um retrato da área refletido em seus artigos. Ciência da Informação, Brasília, v. 30, n. 2, p. 47-63, 2001.

PINHEIRO, Lena Vania Ribeiro. Ciência da Informação: páginas de uma revista em 25 anos. Ciência da Informação, Brasília, v. 25, n. 3, 1996.
; BRÄSCHER, Marisa; BURNIER, Sonia. Ciência da Informação: 32 anos (1972-2004) no caminho da história e horizontes de um periódico científico brasileiro. Ciência da Informação, Brasília, v. 34, n. 3, p. 1-52, 2005.

TRZESNIAK, Piotr. A concepção e a construção da revista científica. In: CURSO de editoração científica, n. 10. Petrópolis: ABEC, 2001. p.17-23.

WHITE, Howard D.; MCCAIN, Katherine W. Bibliometrics. Annual Review of Information Science and Technology, Medford, v. 24, p. 119-186, 1989.

ZANOTTO, Edgar Dutra. A defasagem entre a ciência e a tecnologia nacionais. Pesquisa FAPESP, São Paulo, n. 43, 1999. Opinião. Disponível em: <http://watson.FAPESP.br/nuplitec/patenteA.htm>. Acesso em: 28 nov. 2004. 